\title{
Maximum principles for some quasilinear elliptic systems
}

\author{
Salvatore Leonardi ${ }^{a, *}$, Francesco Leonetti $^{\mathrm{b}}$, Cristina Pignotti $^{\mathrm{b}}$, \\ Eugénio Rocha ${ }^{c}$, Vasile Staicu ${ }^{c}$ \\ ${ }^{\text {a }}$ DMI - Department of Mathematics and Informatics, University of Catania, Viale A. Doria 6, 95125 \\ Catania, Italy \\ b DISIM - Department of Information Engineering, Computer Science and Mathematics, University of \\ L'Aquila, 67100 L'Aquila, Italy \\ ${ }^{\mathrm{c}}$ CIDMA - Center for Research and Development in Mathematics and Applications, Department of \\ Mathematics, University of Aveiro, 3810-193 Aveiro, Portugal
}

\section{A R T I C L E I N F O}

\section{Article history:}

Received 1 November 2018

Accepted 6 November 2018

Communicated by Enzo Mitidieri

\section{$M S C$ :}

$35 \mathrm{~B} 50$

$35 \mathrm{~J} 47$

$35 \mathrm{~J} 62$

$49 \mathrm{~N} 60$

Keywords:

Elliptic system

Maximum principle

$r$-staircase support
A B S T R A C T

We give maximum principles for solutions $u: \Omega \rightarrow \mathbb{R}^{N}$ to a class of quasilinear elliptic systems whose prototype is

$$
-\sum_{i=1}^{n} \frac{\partial}{\partial x_{i}}\left(\sum_{\beta=1}^{N} \sum_{j=1}^{n} a_{i, j}^{\alpha, \beta}(x, u(x)) \frac{\partial u^{\beta}}{\partial x_{j}}(x)\right)=0, \quad x \in \Omega,
$$

where $\alpha \in\{1, \ldots, N\}$ is the equation index and $\Omega$ is an open, bounded subset of $\mathbb{R}^{n}$. We assume that coefficients $a_{i, j}^{\alpha, \beta}(x, y)$ are measurable with respect to $x$, continuous with respect to $y \in \mathbb{R}^{N}$, bounded and elliptic. In vectorial problems, when trying to bound the solution by means of the boundary data, we need to bypass De Giorgi's counterexample by means of some additional structure assumptions on the coefficients $a_{i, j}^{\alpha, \beta}(x, y)$. In this paper, we assume that off-diagonal coefficients $a_{i, j}^{\alpha, \beta}, \alpha \neq \beta$, have support in some staircase set along the diagonal in the $y^{\alpha}, y^{\beta}$ plane.

(C) 2018 Elsevier Ltd. All rights reserved.

\section{Introduction}

We consider the system of $N$ equations

$$
-\sum_{i=1}^{n} \frac{\partial}{\partial x_{i}}\left(\sum_{\beta=1}^{N} \sum_{j=1}^{n} a_{i, j}^{\alpha, \beta}(x, u(x)) \frac{\partial u^{\beta}}{\partial x_{j}}(x)\right)=0, \quad x \in \Omega,
$$

\footnotetext{
* Corresponding author.

E-mail addresses: leonardi@dmi.unict.it (S. Leonardi), leonetti@univaq.it (F. Leonetti), pignotti@univaq.it (C. Pignotti), eugenio@ua.pt (E. Rocha), vasile@ua.pt (V. Staicu).
} 
where $\alpha \in\{1, \ldots, N\}$ is the equation index and $\Omega$ is an open, bounded subset of $\mathbb{R}^{n}$, with $n \geq 2$. Moreover, $u: \Omega \subset \mathbb{R}^{n} \rightarrow \mathbb{R}^{N}$ and $u=\left(u^{1}, \ldots, u^{N}\right)$. Coefficients $a_{i, j}^{\alpha, \beta}(x, y)$ are measurable with respect to $x$, continuous with respect to $y \in \mathbb{R}^{N}$, bounded and elliptic.

In scalar case $(N=1)$ a maximum principle is available as

$$
\sup _{\Omega} u \leq \sup _{\partial \Omega} u .
$$

In vectorial case $(N \geq 2)$, in general, we cannot expect to bound $u$ inside $\Omega$ by means of its boundary values: in [1] it is shown that it can be constructed a system of linear equations with measurable bounded coefficients $a_{i, j}^{\alpha, \beta}(x)$ whose solution is the function $u(x)=x /|x|^{\gamma}$ defined in the unit ball centred at the origin with a suitable $\gamma>1$. Then $u(x)=x$ on the boundary of $\Omega$ but $u$ blows up inside $\Omega$; see also [12,2], the surveys $[9,10]$ and the recent paper [11].

So, the main effort is finding (additional) structure assumptions on coefficients $a_{i, j}^{\alpha, \beta}(x, u)$ that keep away De Giorgi's counterexample and that allow for regularity. A simple case of such a structure is the case in which off-diagonal coefficients $a_{i, j}^{\alpha, \beta}$, with $\beta \neq \alpha$, are identically zero; in such a case, the system (1.1) is (almost) decoupled, since $D_{j} u^{\alpha}=\frac{\partial u^{\alpha}}{\partial x_{j}}$ appears only in the $\alpha$ row of the system

$$
-\sum_{i=1}^{n} \frac{\partial}{\partial x_{i}}\left(\sum_{j=1}^{n} a_{i, j}^{\alpha, \alpha}(x, u(x)) \frac{\partial u^{\alpha}}{\partial x_{j}}(x)\right)=0, \quad x \in \Omega,
$$

then, we can use the maximum principle for one single equation and we get

$$
\sup _{\Omega} u^{\alpha} \leq \sup _{\partial \Omega} u^{\alpha} .
$$

A further step happens when off-diagonal coefficients $a_{i, j}^{\alpha, \beta}(x, u)$, with $\beta \in\{1, \ldots, N\} \backslash\{\alpha\}$, vanish when $u^{\alpha}$ is large, namely,

$$
u^{\alpha}>\theta^{\alpha} \Longrightarrow a_{i, j}^{\alpha, \beta}(x, u)=0 \quad \forall \beta \in\{1, \ldots, N\} \backslash\{\alpha\},
$$

see [12]. In this case, the $\alpha$ row of the system (1.1) becomes decoupled, like (1.3), when $x \in\left\{u^{\alpha}>\theta^{\alpha}\right\}$; then we get

$$
\sup _{\Omega} u^{\alpha} \leq \max \left\{\theta^{\alpha} ; \sup _{\partial \Omega} u^{\alpha}\right\}
$$

see $[12,3]$ and $[7]$.

Actually, what matters is the possibility to suitably control from below the quantity

$$
\sum_{i=1}^{n} \sum_{\beta=1}^{N} \sum_{j=1}^{n} a_{i, j}^{\alpha, \beta}(x, u) D_{j} u^{\beta} D_{i} u^{\alpha}
$$

as follows

$$
u^{\alpha}>\theta^{\alpha} \Longrightarrow \sum_{i=1}^{n} \sum_{\beta=1}^{N} \sum_{j=1}^{n} a_{i, j}^{\alpha, \beta}(x, u) D_{j} u^{\beta} D_{i} u^{\alpha} \geq \nu^{\alpha}\left|D u^{\alpha}\right|^{2}-M^{\alpha},
$$

for a suitable positive constant $\nu^{\alpha}$ and a nonnegative constant $M^{\alpha}$. Such an assumption guarantees the following estimate

$$
\sup _{\Omega} u^{\alpha} \leq \max \left\{\theta^{\alpha} ; \sup _{\partial \Omega} u^{\alpha}\right\}+c\left(\frac{M^{\alpha}}{\nu}\right)^{1 / 2},
$$

for a suitable positive constant $c$ depending only on $n$ and the measure of $\Omega$, see [3].

Since we do not know the solution $u$, condition (1.7) is replaced by the following stronger version

$$
y^{\alpha}>\theta^{\alpha} \Longrightarrow \sum_{i=1}^{n} \sum_{\beta=1}^{N} \sum_{j=1}^{n} a_{i, j}^{\alpha, \beta}(x, y) p_{j}^{\beta} p_{i}^{\alpha} \geq \nu\left|p^{\alpha}\right|^{2}-M^{\alpha},
$$




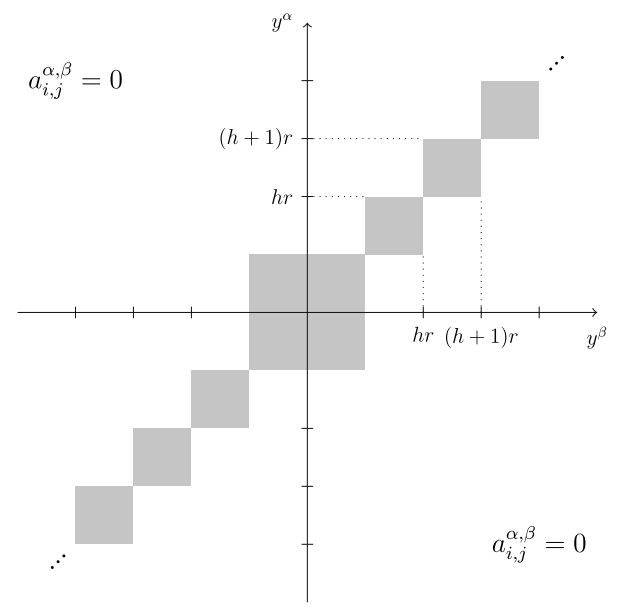

Fig. 1. Support contained in a $r$-staircase set: off-diagonal entries $a_{i, j}^{\alpha, \beta}$ vanish on the white part of the picture; they might be non zero only on the grey part.

for all $y \in \mathbb{R}^{N}$ and all $p \in \mathbb{R}^{N \times n}$. Let us mention that a kind of maximum modulus principle can be found in [8] under the following structure assumption: there exist numbers $\lambda>0, L \geq 0$ and two nonnegative functions $d(x), g(x)$, such that

$$
\begin{aligned}
& \sum_{\alpha=1}^{N} \sum_{\gamma=1}^{N} \frac{y^{\alpha} y^{\gamma}}{|y|^{2}}\left(\sum_{i=1}^{n} p_{i}^{\gamma} \sum_{\beta=1}^{N} \sum_{j=1}^{n} a_{i, j}^{\alpha, \beta}(x, y) p_{j}^{\beta}\right) \\
\geq & -\left\{\delta|p|^{2}+\left(\frac{1}{\delta}\right)^{\lambda}\left[d(x)|y|^{2}+g(x)\right]\right\}
\end{aligned}
$$

is fulfilled for all $\delta \in] 0,1[$ and all $(x, y, p)$, with $|y|>L$.

Note that [8] and [3] deal with systems having a nonlinear dependence on the gradient $D u$.

Going back to condition (1.9) we stress that such an assumption requires that, when we are above the level $\theta^{\alpha}$, the row $\alpha$ has such a good behaviour. In the present paper, we study a different situation: off-diagonal coefficients $a_{i, j}^{\alpha, \beta}$ (that are responsible of the appearance of the other components $D u^{\beta}$ ) appear above every level $\theta^{\alpha}$ but their support is contained in a sequence of squares, see Fig. 1.

Such a condition turned out to be useful when proving existence of solutions to elliptic systems with a right-hand side which is a measure, see [4] and [5].

Under the assumption that the support of the off-diagonal coefficients $a_{i, j}^{\alpha, \beta}$ have such r-staircase shape, we consider the maximum of the boundary values of all components

$$
M:=\max \left\{\sup _{\partial \Omega} u^{1}, \ldots, \sup _{\partial \Omega} u^{N}\right\}
$$

so

$$
h r \leq M<(h+1) r
$$

for some integer $h$; then, we are able to prove that

$$
u^{\alpha} \leq S:=(h+1) r
$$

provided $h \neq-1$, see Fig. 2 (left); when $h=-1$ we have $u^{\alpha} \leq r$, see Fig. 2 (right).

We are able to provide a minimum principle as well. The previous result is true when the support of off-diagonal coefficients $a_{i, j}^{\alpha, \beta}$ is contained in the squares along the diagonal $y^{\alpha}=y^{\beta}$. If we allow the support 

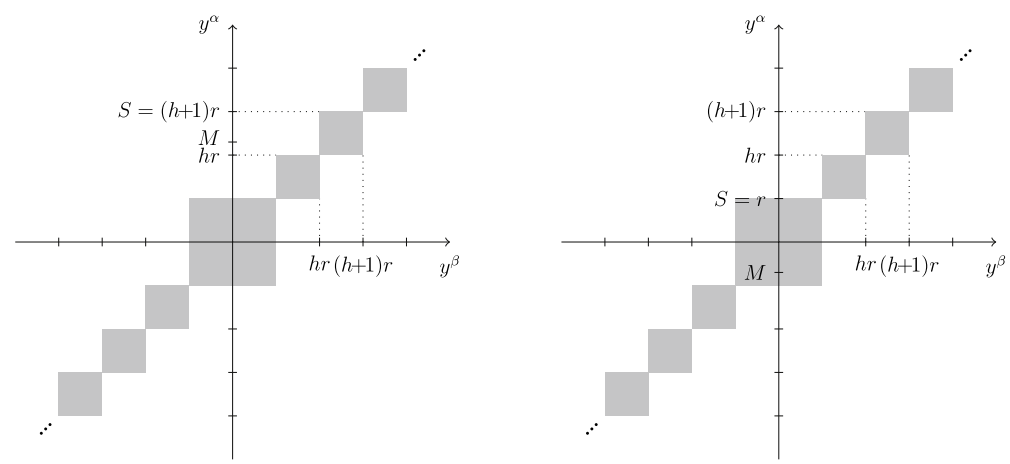

Fig. 2. (left) $h r \leq M<(h+1) r$, with $h \neq-1$; (right) $-r \leq M<0$.

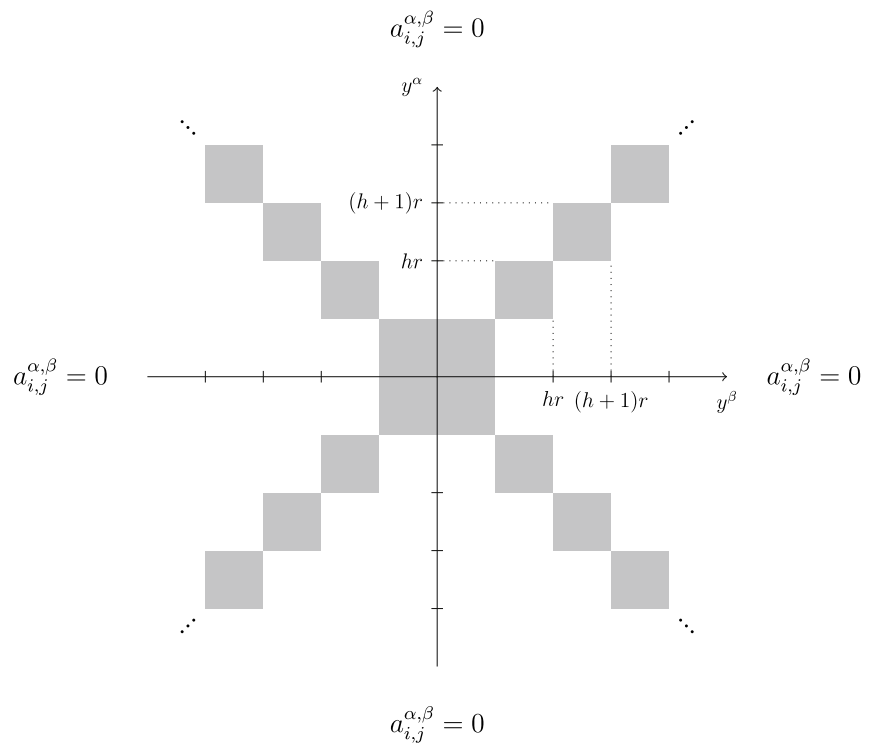

Fig. 3. Support contained in a crossed $r$-staircase set: off-diagonal entries $a_{i, j}^{\alpha, \beta}$ vanish on the white part of the picture; they might be non zero only on the grey part.

to stay also in the other diagonal $y^{\alpha}=-y^{\beta}$, so that the support is contained in a crossed $r$-staircase set as in Fig. 3, then we can prove the maximum modulus estimate

$$
\left|u^{\alpha}\right| \leq S^{*}:=r\left(1+\operatorname{int}\left[\frac{M^{*}}{r}\right]\right)
$$

where

$$
M^{*}:=\max \left\{\sup _{\partial \Omega}\left|u^{1}\right|, \ldots, \sup _{\partial \Omega}\left|u^{N}\right|\right\}
$$

and int $[t]$ denotes the integer part of $t$, that is, the largest integer less than or equal to $t$.

We are also able to give other results assuming a different shape of the support of the off-diagonal coefficients; they apply only to solutions satisfying suitable compatibility conditions.

The paper is organized as follows: in Section 2 we list all the required assumptions and we state the main theorems whose proofs appear in Section 3. An example of a system verifying our assumptions but neither (1.9) nor (1.10) is given in Section 4. 


\section{Assumptions and results}

Assume $\Omega$ is an open bounded subset of $\mathbb{R}^{n}$, with $n \geq 2$. For the sake of brevity, $[k]$ denotes the set $\{1, \ldots, k\}$ when $k \geq 1$ is an integer. Consider the system of $N \geq 2$ equations

$$
-\sum_{i \in[n]} \frac{\partial}{\partial x_{i}}\left(\sum_{\beta \in[N]} \sum_{j \in[n]} a_{i, j}^{\alpha, \beta}(x, u) \frac{\partial}{\partial x_{j}} u^{\beta}\right)=0 \text { in } \Omega, \text { for } \alpha \in[N] .
$$

$(\mathcal{A})$ For all $i, j \in[n]$, and all $\alpha, \beta \in[N]$, we require that $a_{i, j}^{\alpha, \beta}: \Omega \times \mathbb{R}^{N} \rightarrow \mathbb{R}$ satisfies the following conditions:

$\left(\mathcal{A}_{0}\right) x \mapsto a_{i, j}^{\alpha, \beta}(x, y)$ is measurable and $y \mapsto a_{i, j}^{\alpha, \beta}(x, y)$ is continuous;

$\left(\mathcal{A}_{1}\right)$ (boundedness of all the coefficients) for some positive constant $c>0$, we have

$$
\left|a_{i, j}^{\alpha, \beta}(x, y)\right| \leq c
$$

for almost all $x \in \Omega$ and for all $y \in \mathbb{R}^{N}$;

$\left(\mathcal{A}_{2}\right)$ (ellipticity of all the coefficients) for some positive constant $m>0$, we have

$$
\sum_{\alpha, \beta \in[N]} \sum_{i, j \in[n]} a_{i, j}^{\alpha, \beta}(x, y) \xi_{i}^{\alpha} \xi_{j}^{\beta} \geq m|\xi|^{2}
$$

for almost all $x \in \Omega$, for all $y \in \mathbb{R}^{N}$ and for all $\xi \in \mathbb{R}^{N \times n}$;

$\left(\mathcal{A}_{3}\right)$ (r-staircase support of the off-diagonal) there exists $r \in(0,+\infty)$ such that when $\alpha \neq \beta$,

$$
\begin{aligned}
& a_{i, j}^{\alpha, \beta}(x, y) \neq 0 \text { implies either } y \in\left\{\left|y^{\alpha}\right|<r,\left|y^{\beta}\right|<r\right\} \text { or } \\
& y \in \bigcup_{h \in \mathbb{Z}}\left\{h r<y^{\alpha}<(h+1) r, h r<y^{\beta}<(h+1) r\right\}
\end{aligned}
$$

(see Fig. 1).

We say that a function $u: \Omega \rightarrow \mathbb{R}^{N}$ is a weak solution of the system $(2.1)$, if $u \in W^{1,2}\left(\Omega, \mathbb{R}^{N}\right)$ and

$$
\int_{\Omega} \sum_{\alpha, \beta \in[N]} \sum_{i, j \in[n]} a_{i, j}^{\alpha, \beta}(x, u(x)) D_{j} u^{\beta}(x) D_{i} \varphi^{\alpha}(x) d x=0,
$$

for all $\varphi \in W_{0}^{1,2}\left(\Omega, \mathbb{R}^{N}\right)$.

Remark 1. Suppose assumptions $(\mathcal{A})$ hold. Then conditions of Leray-Lions Theorem (see [6]) are satisfied and we get existence of weak solutions to $(2.2)$ provided a boundary datum $u_{*} \in W^{1,2}\left(\Omega, \mathbb{R}^{N}\right)$ has been fixed.

In the aforementioned setting, weak solutions enjoy the following maximum principle.

Theorem 1. Suppose assumptions $(\mathcal{A})$ hold. Then a weak solution u of system (2.1) satisfies

$$
u^{\alpha}(x) \leq S \text { for almost every } x \in \Omega, \quad \text { for all } \alpha \in[N]
$$

where

$$
S:=\left\{\begin{array}{lll}
r\left(1+\operatorname{int}\left[\frac{M}{r}\right]\right) & \text { if } 1+\operatorname{int}\left[\frac{M}{r}\right] \neq 0, \\
r & \text { if } 1+\operatorname{int}\left[\frac{M}{r}\right]=0,
\end{array}\right.
$$




$$
M:=\max \left\{\sup _{\partial \Omega} u^{1}, \ldots, \sup _{\partial \Omega} u^{N}\right\}
$$

Moreover

$$
u^{\alpha}(x) \geq I \text { for almost every } x \in \Omega, \quad \text { for all } \alpha \in[N],
$$

where

$$
I:=\left\{\begin{array}{lll}
-r\left(1+\operatorname{int}\left[\frac{\tilde{M}}{r}\right]\right) & \text { if } & 1+\operatorname{int}\left[\frac{\tilde{M}}{r}\right] \neq 0, \\
-r & \text { if } & 1+\operatorname{int}\left[\frac{\tilde{M}}{r}\right]=0
\end{array}\right.
$$

and

$$
\tilde{M}:=-\min \left\{\inf _{\partial \Omega} u^{1}, \ldots, \inf _{\partial \Omega} u^{N}\right\} .
$$

We now modify the assumption on the support of the off-diagonal coefficients. Namely, we assume $\left(\tilde{\mathcal{A}}_{3}\right)$ (crossed $r$-staircase support of the off-diagonal) there exists $r \in(0,+\infty)$ such that when $\alpha \neq \beta$,

$$
\begin{aligned}
& a_{i, j}^{\alpha, \beta}(x, y) \neq 0 \text { implies either } y \in\left\{\left|y^{\alpha}\right|<r,\left|y^{\beta}\right|<r\right\} \text { or } \\
& y \in \bigcup_{h=1}^{+\infty}\left\{h r<\left|y^{\alpha}\right|<(h+1) r, h r<\left|y^{\beta}\right|<(h+1) r\right\}
\end{aligned}
$$

(see Fig. 3).

If we denote by $(\tilde{\mathcal{A}})$ the set of assumptions $\left(\mathcal{A}_{0}\right),\left(\mathcal{A}_{1}\right),\left(\mathcal{A}_{2}\right),\left(\tilde{\mathcal{A}}_{3}\right)$ we can prove the following companion of Theorem 1.

Theorem 2. Suppose assumptions $(\tilde{\mathcal{A}})$ hold. Then a weak solution u of system (2.1) satisfies

$$
\left|u^{\alpha}(x)\right| \leq S^{*} \quad \text { for almost every } x \in \Omega, \quad \text { for all } \alpha \in[N]
$$

where

$$
\begin{gathered}
S^{*}:=r\left(1+\operatorname{int}\left[\frac{M^{*}}{r}\right]\right) \\
M^{*}:=\max \left\{\sup _{\partial \Omega}\left|u^{1}\right|, \ldots, \sup _{\partial \Omega}\left|u^{N}\right|\right\} .
\end{gathered}
$$

Now, we can give other results assuming a different shape of support for the off-diagonal coefficients. However, they apply only to solutions satisfying suitable compatibility conditions. We assume,

$\left(\mathcal{A}_{3}^{*}\right)$ For fixed $\left(L_{+}^{1}, \ldots, L_{+}^{N}\right) \in \mathbb{R}^{N}$, if $\alpha \neq \beta$, then

$$
a_{i, j}^{\alpha, \beta}(x, y) \neq 0 \text { and } y^{\alpha}>L_{+}^{\alpha} \Longrightarrow y^{\beta}>L_{+}^{\beta},
$$

(see Fig. 4).

Let us denote by $\left(\mathcal{A}^{*}\right)$ the set of assumptions $\left(\mathcal{A}_{0}\right),\left(\mathcal{A}_{1}\right),\left(\mathcal{A}_{2}\right),\left(\mathcal{A}_{3}^{*}\right)$.

Theorem 3. Suppose assumptions $\left(\mathcal{A}^{*}\right)$ hold. Then a weak solution u of system (2.1) with

$$
\sup _{\partial \Omega} u^{\alpha} \leq L_{+}^{\alpha}, \quad \forall \alpha \in[N]
$$

satisfies

$$
u^{\alpha}(x) \leq L_{+}^{\alpha} \quad \text { for a.e. } \quad x \in \Omega, \forall \alpha \in[N] .
$$




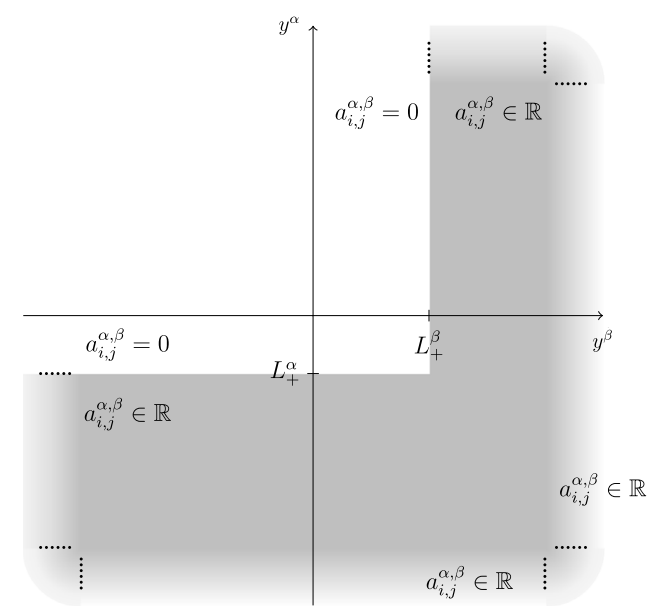

Fig. 4. Support verifying $\mathcal{A}_{3}^{*}$ : off-diagonal entries $a_{i, j}^{\alpha, \beta}$ vanish on the white part of the picture; they might be non zero only on the grey part.

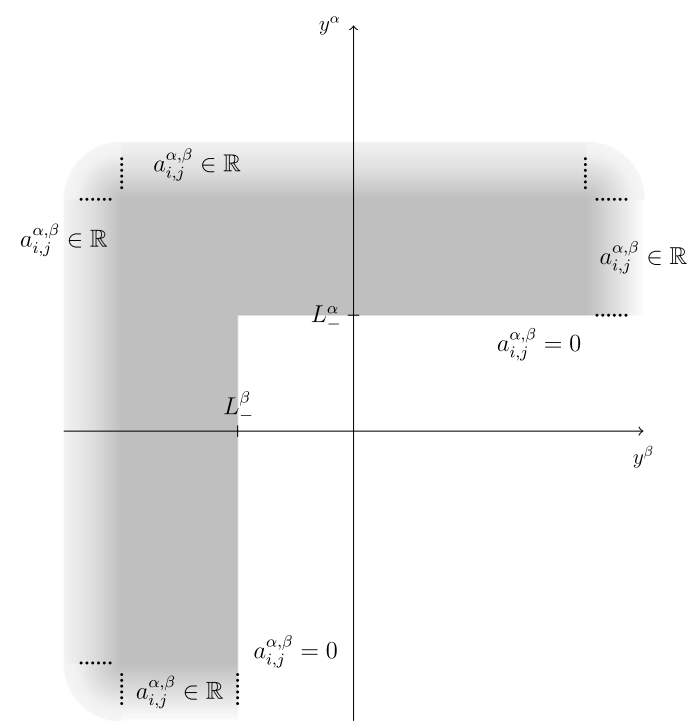

Fig. 5. Support verifying $\mathcal{A}_{3}^{* *}$ : off-diagonal entries $a_{i, j}^{\alpha, \beta}$ vanish on the white part of the picture; they might be non zero only on the grey part.

Remark 2. Note that (2.12) represents a compatibility condition between the boundary values of $u^{\alpha}$ and the supports of off-diagonal coefficients $a_{i, j}^{\alpha, \beta}$.

In the same spirit, one can give a minimum principle, under a compatibility condition on the boundary datum, by assuming the following

$\left(\mathcal{A}_{3}^{* *}\right)$ For fixed $\left(L_{-}^{1}, \ldots, L_{-}^{N}\right) \in \mathbb{R}^{N}$, if $\alpha \neq \beta$, then

$$
a_{i, j}^{\alpha, \beta}(x, y) \neq 0 \quad \text { and } \quad y^{\alpha}<L_{-}^{\alpha} \quad \Longrightarrow y^{\beta}<L_{-}^{\beta},
$$

(see Fig. 5).

Let us denote $\left(\mathcal{A}^{* *}\right)$ the set of assumptions $\left(\mathcal{A}_{0}\right),\left(\mathcal{A}_{1}\right),\left(\mathcal{A}_{2}\right),\left(\mathcal{A}_{3}^{* *}\right)$. 


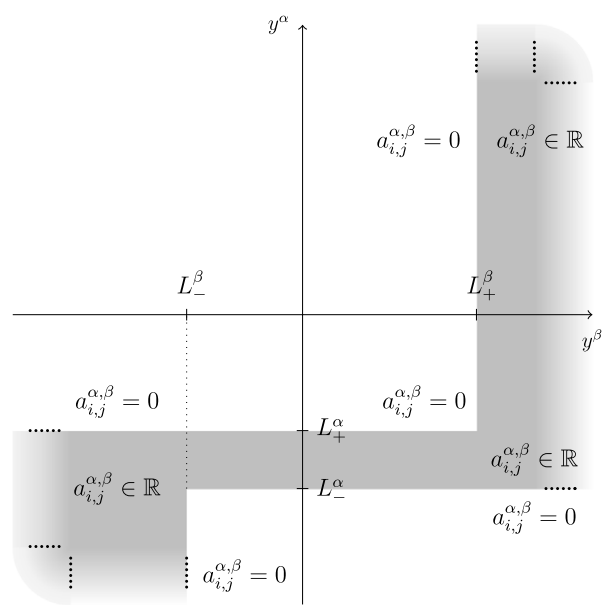

Fig. 6. Support verifying $\mathcal{A}_{3}^{*}$ and $\mathcal{A}_{3}^{* *}$ : off-diagonal entries $a_{i, j}^{\alpha, \beta}$ vanish on the white part of the picture; they might be non zero only on the grey part.

Theorem 4. Suppose assumptions $\left(\mathcal{A}^{* *}\right)$ hold. Then a weak solution $u$ of system (2.1) with

$$
\inf _{\partial \Omega} u^{\alpha} \geq L_{-}^{\alpha}, \quad \forall \alpha \in[N]
$$

satisfies

$$
u^{\alpha}(x) \geq L_{-}^{\alpha} \quad \text { for a.e. } x \in \Omega, \forall \alpha \in[N] \text {. }
$$

Corollary 1. Let $\left(L_{+}^{1}, \ldots, L_{+}^{N}\right)$ and $\left(L_{-}^{1}, \ldots, L_{-}^{N}\right)$ be fixed vectors in $\mathbb{R}^{N}$ with $L_{-}^{\alpha} \leq L_{+}^{\alpha}, \forall \alpha \in[N]$. Suppose assumptions $\left(\mathcal{A}^{*}\right)$ and $\left(\mathcal{A}_{3}^{* *}\right)$ hold, see Fig. 6. Then a weak solution u of system (2.1) with

$$
L_{-}^{\alpha} \leq u^{\alpha} \leq L_{+}^{\alpha} \quad \text { on } \partial \Omega, \forall \alpha \in[N]
$$

satisfies

$$
L_{-}^{\alpha} \leq u^{\alpha} \leq L_{+}^{\alpha} \quad \text { in } \quad \Omega, \forall \alpha \in[N]
$$

Corollary 2. Suppose assumptions $\left(\mathcal{A}^{*}\right)$ and $\left(\mathcal{A}_{3}^{* *}\right)$ hold with $L_{+}^{\alpha}=L_{-}^{\alpha}=0$, see Fig. 7. Then a weak solution $u$ of system (2.1) with

$$
u^{\alpha}=0 \quad \text { on } \partial \Omega, \forall \alpha \in[N] \text {, }
$$

satisfies

$$
u^{\alpha}=0 \quad \text { in } \quad \Omega, \forall \alpha \in[N]
$$

\section{Proofs of the results}

Proof of Theorem 1. Let $u \in W^{1,2}\left(\Omega, \mathbb{R}^{N}\right)$ be a weak solution of system (2.1). Let $L \in \mathbb{R}$ be such that

$$
\sup _{\partial \Omega} u^{\alpha} \leq L \quad \text { for all } \alpha \in[N] .
$$

Consider the test function

$$
\varphi^{\alpha}(x):=\max \left\{0, u^{\alpha}(x)-L\right\}, \quad \text { for all } \alpha \in[N]
$$




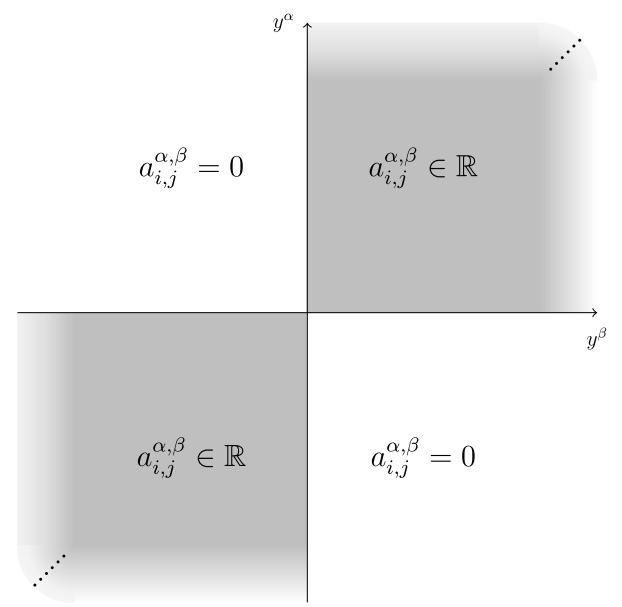

Fig. 7. Support verifying $\mathcal{A}_{3}^{*}$ and $\mathcal{A}_{3}^{* *}$ with $L_{+}^{\alpha}=L_{-}^{\alpha}=0$ : off-diagonal entries $a_{i, j}^{\alpha, \beta}$ vanish on the white part of the picture; they might be non zero only on the grey part.

Then

$$
D_{i} \varphi^{\alpha}=\mathbf{1}_{\left\{u^{\alpha}>L\right\}} D_{i} u^{\alpha} \quad \text { for all } i \in[n] \text { and } \alpha \in[N] .
$$

Using the test function $\varphi$ in the weak form (2.2) of system (2.1), we have

$$
\begin{aligned}
0= & \int_{\Omega} \sum_{\alpha, \beta \in[N]} \sum_{i, j \in[n]} a_{i, j}^{\alpha, \beta} D_{j} u^{\beta} D_{i} \varphi^{\alpha} d x \\
& =\int_{\Omega} \sum_{\alpha, \beta \in[N]} \sum_{i, j \in[n]} a_{i, j}^{\alpha, \beta} D_{j} u^{\beta} \mathbf{1}_{\left\{u^{\alpha}>L\right\}} D_{i} u^{\alpha} d x .
\end{aligned}
$$

Now, the $r$-staircase assumption $\left(\mathcal{A}_{3}\right)$ guarantees that

$$
a_{i, j}^{\alpha, \beta}(x, u(x)) \mathbf{1}_{\left\{u^{\alpha}>L\right\}}(x)=a_{i, j}^{\alpha, \beta}(x, u(x)) \mathbf{1}_{\left\{u^{\beta}>L\right\}}(x) \mathbf{1}_{\left\{u^{\alpha}>L\right\}}(x)
$$

when $\beta \neq \alpha$ and $L / r \in \mathbb{Z} \backslash\{0\}$. It is worthwhile to note that (3.1) holds true when $\alpha=\beta$ as well; then

$$
\begin{aligned}
& \int_{\Omega} \sum_{\alpha, \beta \in[N]} \sum_{i, j \in[n]} a_{i, j}^{\alpha, \beta} D_{j} u^{\beta} \mathbf{1}_{\left\{u^{\alpha}>L\right\}} D_{i} u^{\alpha} d x \\
& =\int_{\Omega} \sum_{\alpha, \beta \in[N]} \sum_{i, j \in[n]} a_{i, j}^{\alpha, \beta} D_{j} u^{\beta} \mathbf{1}_{\left\{u^{\beta}>L\right\}} \mathbf{1}_{\left\{u^{\alpha}>L\right\}} D_{i} u^{\alpha} d x \\
& =\int_{\Omega} \sum_{\alpha, \beta \in[N]} \sum_{i, j \in[n]} a_{i, j}^{\alpha, \beta} D_{j} \varphi^{\beta} D_{i} \varphi^{\alpha} d x .
\end{aligned}
$$

Now we can use the ellipticity assumption $\left(\mathcal{A}_{3}\right)$ with $\xi_{i}^{\alpha}=D_{i} \varphi^{\alpha}$ and we get

$$
m \int_{\Omega} \sum_{\alpha \in[N]}\left|D \varphi^{\alpha}\right|^{2} d x \leq \int_{\Omega} \sum_{\alpha, \beta \in[N]} \sum_{i, j \in[n]} a_{i, j}^{\alpha, \beta} D_{j} \varphi^{\beta} D_{i} \varphi^{\alpha} d x=0 .
$$

This means that

$$
\int_{\Omega} \sum_{\alpha \in[N]}\left|D \varphi^{\alpha}\right|^{2} d x=0
$$

and, since $\varphi^{\alpha} \in W_{0}^{1,2}(\Omega)$, we can use Poincaré inequality

$$
\int_{\Omega}\left|\varphi^{\alpha}\right|^{2} d x \leq C \int_{\Omega}\left|D \varphi^{\alpha}\right|^{2} d x=0
$$


obtaining $\max \left\{0, u^{\alpha}-L\right\}=\varphi^{\alpha}=0$ almost everywhere in $\Omega$, so

$$
u^{\alpha} \leq L \quad \text { almost everywhere in } \Omega .
$$

We now select a suitable value for $L$. We take $L=S$ given by (2.4) and we get (2.3).

Let $\tilde{u}=-u$. Then $\tilde{u} \in W^{1,2}\left(\Omega ; \mathbb{R}^{N}\right)$ and, since $u$ satisfies $(2.2)$, then $\tilde{u}$ verifies

$$
0=\int_{\Omega} \sum_{\alpha, \beta \in[N]} \sum_{i, j \in[n]} \tilde{a}_{i, j}^{\alpha, \beta}(x, \tilde{u}(x)) D_{j} \tilde{u}^{\beta}(x) D_{i} \varphi^{\alpha}(x) d x
$$

for every $\varphi \in W_{0}^{1,2}\left(\Omega, \mathbb{R}^{N}\right)$, where

$$
\tilde{a}_{i, j}^{\alpha, \beta}(x, y):=a_{i, j}^{\alpha, \beta}(x,-y) .
$$

We observe that the new coefficients defined by $(3.3)$ readily satisfy conditions $\left(\mathcal{A}_{0}\right),\left(\mathcal{A}_{1}\right),\left(\mathcal{A}_{2}\right)$.

Moreover, if $\alpha \neq \beta$ and $\tilde{a}_{i, j}^{\alpha, \beta}(x, y) \neq 0$ then, by $\left(\mathcal{A}_{3}\right)$,

$$
\begin{aligned}
& a_{i, j}^{\alpha, \beta}(x,-y) \neq 0 \text { as either }-y \in\left\{\left|y^{\alpha}\right|<r,\left|y^{\beta}\right|<r\right\} \text { or } \\
& -y \in \bigcup_{h \in \mathbb{Z}}\left\{h r<y^{\alpha}<(h+1) r, h r<y^{\beta}<(h+1) r\right\} .
\end{aligned}
$$

Therefore either $y \in\left\{\left|y^{\alpha}\right|<r,\left|y^{\beta}\right|<r\right\}$ or

$$
\exists \tilde{h} \in \mathbb{Z}: y \in\left\{\tilde{h} r<y^{\alpha}<(\tilde{h}+1) r, \tilde{h} r<y^{\beta}<(\tilde{h}+1) r\right\}
$$

and we can conclude that $\tilde{a}_{i, j}^{\alpha, \beta}(x, y)$ satisfy $\left(\mathcal{A}_{3}\right)$ as well. By $(2.3)$ applied to $\tilde{u}=-u$ we deduce that $\tilde{u}^{\alpha} \leq \tilde{S}$ where

$$
\tilde{S}:= \begin{cases}r\left(1+\operatorname{int}\left[\frac{\tilde{M}}{r}\right]\right) & \text { if } 1+\operatorname{int}\left[\frac{\tilde{M}}{r}\right] \neq 0, \\ r & \text { if } 1+\operatorname{int}\left[\frac{\tilde{M}}{r}\right]=0\end{cases}
$$

and

$$
\tilde{M}:=\max \left\{\sup _{\partial \Omega} \tilde{u}^{1}, \ldots, \sup _{\partial \Omega} \tilde{u}^{N}\right\}
$$

Note that

$$
\begin{aligned}
\tilde{M} & =\max \left\{\sup _{\partial \Omega}\left(-u^{1}\right), \ldots, \sup _{\partial \Omega}\left(-u^{N}\right)\right\}=\max \left\{-\inf _{\partial \Omega} u^{1}, \ldots,-\inf _{\partial \Omega} u^{N}\right\} \\
& =-\min \left\{\inf _{\partial \Omega} u^{1}, \ldots, \inf _{\partial \Omega} u^{N}\right\}
\end{aligned}
$$

so that

$$
u^{\alpha} \geq-\tilde{S}=\left\{\begin{array}{lll}
-r\left(1+\operatorname{int}\left[\frac{\tilde{M}}{r}\right]\right) & \text { if } & 1+\operatorname{int}\left[\frac{\tilde{M}}{r}\right] \neq 0, \\
-r & \text { if } & 1+\operatorname{int}\left[\frac{\tilde{M}}{r}\right]=0
\end{array}\right.
$$

and (2.6) is proved too. This ends the proof of Theorem 1.

Proof of Theorem 2. Let $u \in W^{1,2}\left(\Omega, \mathbb{R}^{N}\right)$ be a weak solution of system (2.1). Let $L \in \mathbb{R}$ be such that $\sup _{\partial \Omega}\left|u^{\alpha}\right| \leq L \quad$ for all $\alpha \in[N]$ 
Consider the test function

$$
\tilde{\varphi}^{\alpha}(x):=\max \left\{0, u^{\alpha}(x)-L\right\}+\min \left\{0, u^{\alpha}(x)+L\right\}, \quad \text { for all } \alpha \in[N] .
$$

Then

$$
D_{i} \tilde{\varphi}^{\alpha}=\mathbf{1}_{\left\{\left|u^{\alpha}\right|>L\right\}} D_{i} u^{\alpha} \quad \text { for all } i \in[n] \text { and } \alpha \in[N] .
$$

Using the test function $\tilde{\varphi}$ in the weak form (2.2) of system (2.1), we have

$$
\begin{aligned}
0= & \int_{\Omega} \sum_{\alpha, \beta \in[N]} \sum_{i, j \in[n]} a_{i, j}^{\alpha, \beta} D_{j} u^{\beta} D_{i} \tilde{\varphi}^{\alpha} d x \\
& =\int_{\Omega} \sum_{\alpha, \beta \in[N]} \sum_{i, j \in[n]} a_{i, j}^{\alpha, \beta} D_{j} u^{\beta} \mathbf{1}_{\left\{\left|u^{\alpha}\right|>L\right\}} D_{i} u^{\alpha} d x .
\end{aligned}
$$

Now, the crossed $r$-staircase assumption $\left(\tilde{\mathcal{A}}_{3}\right)$ guarantees that

$$
a_{i, j}^{\alpha, \beta}(x, u(x)) \mathbf{1}_{\left\{\left|u^{\alpha}\right|>L\right\}}(x)=a_{i, j}^{\alpha, \beta}(x, u(x)) \mathbf{1}_{\left\{\left|u^{\beta}\right|>L\right\}}(x) \mathbf{1}_{\left\{\left|u^{\alpha}\right|>L\right\}}(x)
$$

when $\beta \neq \alpha$ and $L / r \in\{1,2, \ldots\}$. Note that (3.4) holds true when $\beta=\alpha$ as well; then

$$
\begin{aligned}
& \int_{\Omega} \sum_{\alpha, \beta \in[N]} \sum_{i, j \in[n]} a_{i, j}^{\alpha, \beta} D_{j} u^{\beta} \mathbf{1}_{\left\{\left|u^{\alpha}\right|>L\right\}} D_{i} u^{\alpha} d x \\
& =\int_{\Omega} \sum_{\alpha, \beta \in[N]} \sum_{i, j \in[n]} a_{i, j}^{\alpha, \beta} D_{j} u^{\beta} \mathbf{1}_{\left\{\left|u^{\beta}\right|>L\right\}} \mathbf{1}_{\left\{u^{\alpha}>L\right\}} D_{i} u^{\alpha} d x \\
& =\int_{\Omega} \sum_{\alpha, \beta \in[N]} \sum_{i, j \in[n]} a_{i, j}^{\alpha, \beta} D_{j} \tilde{\varphi}^{\beta} D_{i} \tilde{\varphi}^{\alpha} d x .
\end{aligned}
$$

As in the proof of Theorem 1, we can use the ellipticity assumption $\left(\mathcal{A}_{3}\right)$ with $\xi_{i}^{\alpha}=D_{i} \tilde{\varphi}^{\alpha}$ and we get $\left|D \tilde{\varphi}^{\alpha}\right|=$ 0 ; since $\tilde{\varphi}^{\alpha} \in W_{0}^{1,2}(\Omega)$, we use Poincaré inequality and we obtain $\max \left\{0, u^{\alpha}-L\right\}+\min \left\{0, u^{\alpha}+L\right\}=\tilde{\varphi}^{\alpha}=0$, so

$$
\left|u^{\alpha}\right| \leq L \quad \text { almost everywhere in } \Omega .
$$

Now we have to select a suitable value for $L$. We take $L=S^{*}$ given by (2.10) and we get (2.9). This ends the proof of Theorem 2 .

Proof of Theorem 3. Let us consider the test function $\varphi$ such that

$$
\varphi^{\alpha}(x):=\max \left\{0, u^{\alpha}(x)-L_{+}^{\alpha}\right\}, \quad \text { for all } \alpha \in[N] .
$$

Since (2.12) holds true, then $\varphi^{\alpha} \in W_{0}^{1,2}(\Omega)$ and

$$
D_{i} \varphi^{\alpha}=\mathbf{1}_{\left\{u^{\alpha}>L_{+}^{\alpha}\right\}} D_{i} u^{\alpha} \quad \text { for all } i \in[n] \text { and } \alpha \in[N] .
$$

Using the test function $\varphi$ in the weak form (2.2) of system (2.1), we have

$$
\begin{aligned}
0= & \int_{\Omega} \sum_{\alpha, \beta \in[N]} \sum_{i, j \in[n]} a_{i, j}^{\alpha, \beta} D_{j} u^{\beta} D_{i} \varphi^{\alpha} d x \\
& =\int_{\Omega} \sum_{\alpha, \beta \in[N]} \sum_{i, j \in[n]} a_{i, j}^{\alpha, \beta} D_{j} u^{\beta} \mathbf{1}_{\left\{u^{\alpha}>L_{+}^{\alpha}\right\}} D_{i} u^{\alpha} d x .
\end{aligned}
$$


Now, assumption $\left(\mathcal{A}_{3}^{*}\right)$ guarantees that

$$
a_{i, j}^{\alpha, \beta}(x, u(x)) \mathbf{1}_{\left\{u^{\alpha}>L_{+}^{\alpha}\right\}}(x)=a_{i, j}^{\alpha, \beta}(x, u(x)) \mathbf{1}_{\left\{u^{\alpha}>L_{+}^{\alpha}\right\}}(x) \mathbf{1}_{\left\{u^{\beta}>L_{+}^{\beta}\right\}}(x)
$$

when $\beta \neq \alpha$. Note that (3.5) holds true when $\beta=\alpha$ as well. Then,

$$
\begin{aligned}
& \int_{\Omega} \sum_{\alpha, \beta \in[N]} \sum_{i, j \in[n]} a_{i, j}^{\alpha, \beta} D_{j} u^{\beta} \mathbf{1}_{\left\{u^{\alpha}>L_{+}^{\alpha}\right\}} D_{i} u^{\alpha} d x \\
& =\int_{\Omega} \sum_{\alpha, \beta \in[N]} \sum_{i, j \in[n]} a_{i, j}^{\alpha, \beta} D_{j} u^{\beta} \mathbf{1}_{\left\{u^{\alpha}>L_{+}^{\alpha}\right\}} \mathbf{1}_{\left\{u^{\beta}>L_{+}^{\beta}\right\}} D_{i} u^{\alpha} d x \\
& =\int_{\Omega} \sum_{\alpha, \beta \in[N]} \sum_{i, j \in[n]} a_{i, j}^{\alpha, \beta} D_{j} \varphi^{\beta} D_{i} \varphi^{\alpha} d x .
\end{aligned}
$$

Now, as in Theorem 1, we can use the ellipticity assumption $\left(\mathcal{A}_{3}\right)$ with $\xi_{i}^{\alpha}=D_{i} \varphi^{\alpha}$ and we get $\left|D \varphi^{\alpha}\right|=0$; since $\varphi^{\alpha} \in W_{0}^{1,2}(\Omega)$, we can use Poincaré inequality and we obtain $\max \left\{0, u^{\alpha}-L_{+}^{\alpha}\right\}=\varphi^{\alpha}=0$, so

$$
u^{\alpha} \leq L_{+}^{\alpha} \quad \text { almost everywhere in } \Omega .
$$

This ends the proof of Theorem 3.

Proof of Theorem 4. Let $\tilde{u}=-u$. Then $\tilde{u} \in W^{1,2}\left(\Omega ; \mathbb{R}^{N}\right)$ and since $u$ satisfies $(2.2)$ then $\tilde{u}$ verifies

$$
0=\int_{\Omega} \sum_{\alpha, \beta \in[N]} \sum_{i, j \in[n]} \tilde{a}_{i, j}^{\alpha, \beta}(x, \tilde{u}(x)) D_{j} \tilde{u}^{\beta}(x) D_{i} \varphi^{\alpha}(x) d x
$$

for every $\varphi \in W_{0}^{1,2}\left(\Omega, \mathbb{R}^{N}\right)$, where

$$
\tilde{a}_{i, j}^{\alpha, \beta}(x, y):=a_{i, j}^{\alpha, \beta}(x,-y)
$$

We observe that new coefficients defined by (3.7) readily satisfy conditions $\left(\mathcal{A}_{0}\right),\left(\mathcal{A}_{1}\right),\left(\mathcal{A}_{2}\right)$. Moreover, if $\alpha \neq \beta$ and $\tilde{a}_{i, j}^{\alpha, \beta}(x, y) \neq 0$ then, by $\left(\mathcal{A}_{3}^{* *}\right)$, we deduce that for $-y^{\alpha}<L_{-}^{\alpha}$ it must be $-y^{\beta}<L_{-}^{\beta}$. Thus, if $\tilde{a}_{i, j}^{\alpha, \beta}(x, y) \neq 0$ and $y^{\alpha}>-L_{-}^{\alpha}$, then $y^{\beta}>-L_{-}^{\beta}$. Therefore, we can conclude that $\tilde{a}_{i, j}^{\alpha, \beta}(x, y)$ satisfy $\left(\mathcal{A}_{3}^{*}\right)$ with $L_{+}^{\alpha}=-L_{-}^{\alpha}$, for $\alpha \in[N]$. By (2.13) applied to $\tilde{u}=-u$ we deduce that

$$
\tilde{u}^{\alpha} \leq-L_{-}^{\alpha} \quad \text { almost everywhere in } \Omega
$$

and then the claim is proved. This ends the proof of Theorem 4.

\section{An example}

In this section we provide an example satisfying our structural conditions $(\mathcal{A})$ but verifying neither $(1.10)$ nor (1.9). We fix $n=3, N=2$ and we set

$$
\begin{aligned}
& a_{i, j}^{1,1}(x, y)=a_{i, j}^{2,2}(x, y)=\delta_{i j}, \\
& a_{i, j}^{1,2}(x, y)=w(y) \delta_{i, j}, \quad a_{i, j}^{2,1}(x, y)=-a_{j, i}^{1,2}(x, y),
\end{aligned}
$$

where $\delta_{i j}$ is Kronecker delta, $w(y) \geq 0$ is a bounded, continuous function, with support contained in the $r$-staircase set, see Fig. 1. Furthermore, we require that $w=5$ in the centre of every square of the sector $y^{1} \geq r, y^{2} \geq r$, that is

$$
w((h+1 / 2) r,(h+1 / 2) r)=5,
$$

for every $h \in \mathbb{N}$. Note that

$$
a^{1,1}=a^{2,2}=\left(\begin{array}{lll}
1 & 0 & 0 \\
0 & 1 & 0 \\
0 & 0 & 1
\end{array}\right) \quad a^{1,2}=\left(\begin{array}{lll}
w(y) & 0 & 0 \\
0 & w(y) & 0 \\
0 & 0 & w(y)
\end{array}\right)=-a^{2,1} .
$$

Observe that the coefficients defined in (4.1) readily satisfy conditions $(\mathcal{A})$. 


\subsection{Comparison with condition (1.10)}

Let us recall condition (1.10): there exist numbers $\lambda>0, L \geq 0$ and two nonnegative functions $d(x), g(x)$, such that

$$
\begin{gathered}
I:=\sum_{\alpha \in[N]} \sum_{\gamma \in[N]} \frac{y^{\alpha} y^{\gamma}}{|y|^{2}}\left(\sum_{i \in[n]} p_{i}^{\gamma} \sum_{\beta \in[N]} \sum_{j \in[n]} a_{i, j}^{\alpha, \beta}(x, y) p_{j}^{\beta}\right) \\
\geq-\left\{\delta|p|^{2}+\left(\frac{1}{\delta}\right)^{\lambda}\left[d(x)|y|^{2}+g(x)\right]\right\}:=Q
\end{gathered}
$$

is fulfilled for all $\delta \in] 0,1[$ and all $(x, y, p)$, with $|y|>L$.

We want to show that the coefficients defined in (4.1) do not satisfy (4.3). To this purpose we choose

$$
\begin{aligned}
& y^{1}=y^{2}>0, \\
& p_{1}^{2}=p_{2}^{1}=p_{2}^{2}=p_{3}^{1}=p_{3}^{2}=0, \quad p_{1}^{1} \neq 0
\end{aligned}
$$

and we replace in (4.3). According to the aforementioned definitions, we can rewrite

$$
I=\frac{1}{2}\left(p_{1}^{1}\right)^{2}(1-w(y)),
$$

while

$$
Q=-\delta\left(p_{1}^{1}\right)^{2}-\left(\frac{1}{\delta}\right)^{\lambda}\left[2 d(x)\left(y^{1}\right)^{2}+g(x)\right]
$$

On the other hand

$$
2 d(x)\left(y^{1}\right)^{2}+g(x) \leq[2 d(x)+g(x)+1]\left[1+\left(y^{1}\right)^{2}\right] .
$$

We select $y$ to be the centre of the squares of the sector $y^{1} \geq r, y^{2} \geq r$ :

$$
y^{1}=y^{2}=\left(h+\frac{1}{2}\right) r
$$

with $h \in \mathbb{N}$ and $h \geq \operatorname{int}\left[-\frac{1}{2}+\frac{L}{r \sqrt{2}}\right]+1$ in such a way that $|y|>L$ and

$$
w(y)=w\left(y^{1}, y^{1}\right)=5
$$

Moreover, from (4.5) we get

$$
I=-2\left(p_{1}^{1}\right)^{2}
$$

and from (4.7) we deduce

$$
2 d(x)\left(y^{1}\right)^{2}+g(x) \leq 4[2 d(x)+g(x)+1]\left(1+r^{2}\right) h^{2} .
$$

Merging (4.10) into (4.6) and using (4.9), for any $\delta \in] 0,1[$, we obtain

$$
\begin{aligned}
Q & \geq-\delta\left(p_{1}^{1}\right)^{2}-4\left(\frac{1}{\delta}\right)^{\lambda}[2 d(x)+g(x)+1]\left(1+r^{2}\right) h^{2} \\
& =I+(2-\delta)\left(p_{1}^{1}\right)^{2}-4\left(\frac{1}{\delta}\right)^{\lambda}[2 d(x)+g(x)+1]\left(1+r^{2}\right) h^{2} \\
& \geq I+\left(p_{1}^{1}\right)^{2}-4\left(\frac{1}{\delta}\right)^{\lambda}[2 d(x)+g(x)+1]\left(1+r^{2}\right) h^{2}, \quad \forall p_{1}^{1} \neq 0 .
\end{aligned}
$$

So that, fixed any $x \in \Omega$ and choosing

$$
p_{1}^{1}=3 h \sqrt{\left(\frac{1}{\delta}\right)^{\lambda}[2 d(x)+g(x)+1]\left(1+r^{2}\right)},
$$

we immediately deduce

$$
Q>I
$$




\subsection{Comparison with condition (1.9)}

Let us recall condition (1.9): for every $\alpha \in\{1,2, \ldots, N\}$ there exist $\theta^{\alpha} \in \mathbb{R}, M^{\alpha} \geq 0$ and $\nu^{\alpha}>0$ such that

$$
\begin{aligned}
y^{\alpha}>\theta^{\alpha} & \Rightarrow \quad I^{\alpha}:=\sum_{i \in[n]} \sum_{\beta \in[N]} \sum_{j \in[n]} a_{i, j}^{\alpha, \beta}(x, y) p_{j}^{\beta} p_{i}^{\alpha} \\
& \geq \nu^{\alpha}\left|p^{\alpha}\right|^{2}-M^{\alpha}:=Q^{\alpha}
\end{aligned}
$$

We want to prove that coefficients (4.1) do not satisfy (4.12) when $\alpha=2$. As before, we choose $y$ to be the centre of the squares but we change a little bit the matrix $p$ :

$$
\begin{aligned}
& p_{2}^{1}=p_{2}^{2}=p_{3}^{1}=p_{3}^{2}=0, \quad p_{1}^{2}=1, \quad p_{1}^{1} \neq 0 \\
& y^{1}=y^{2}=\left(h+\frac{1}{2}\right) r
\end{aligned}
$$

with $h \in \mathbb{N}$ and $h \geq \operatorname{int}\left[\theta^{2} / r-1 / 2\right]+1$, so that $y^{2}>\theta^{2}$. We then replace in (4.12). According to the aforementioned definitions and recalling that

$$
w(y)=w\left(y^{1}, y^{2}\right)=5,
$$

we can rewrite

$$
\begin{aligned}
I^{2} & =-w(y) p_{1}^{1}+1=-5 p_{1}^{1}+1 \\
Q^{2} & =\nu^{2}-M^{2}
\end{aligned}
$$

This, for $p_{1}^{1}$ sufficiently large, implies that

$$
I^{2}<Q^{2}
$$

\section{Acknowledgements}

The first author has been supported by Piano della Ricerca 2016-2018-linea di intervento 2: "Metodi variazionali ed equazioni differenziali", GNAMPA and UNIVAQ. The second and third authors gladly acknowledge the support of GNAMPA, INdAM and UNIVAQ. The last two authors acknowledge partial support by the Portuguese Foundation for Science and Technology (FCT), through CIDMA - Center for Research and Development in Mathematics and Applications, within project UID/MAT/04106/2013.

\section{References}

[1] E. De Giorgi, Un esempio di estremali discontinue per un problema variazionale di tipo ellittico, Boll. Unione Mat. Ital. (4) 1 (1968) 135-137.

[2] S. Leonardi, On Constants of some regularity theorems. De Giorgi's type counterexample, Math. Nachr. 192 (1998) 191-204.

[3] F. Leonetti, P.V. Petricca, Regularity for solutions to some nonlinear elliptic systems, Complex Var. Elliptic Equ. 56 (12) (2011) 1099-1113.

[4] F. Leonetti, E. Rocha, V. Staicu, Quasilinear elliptic systems with measure data, Nonlinear Anal. 154 (2017) $210-224$.

[5] F. Leonetti, E. Rocha, V. Staicu, Smallness and cancellation in some elliptic systems with measure data, J. Math. Anal. Appl. 465 (2018) 885-902.

[6] J. Leray, J.L. Lions, Quelques résultats de Višik sur les problèmes elliptiques non linéaires par les méthodes de Minty-Browder, Bull. Soc. Math. Fr. 93 (1965) 97-107.

[7] F. Mandras, Principio di massimo per una classe di sistemi ellittici degeneri quasi lineari, Rend. Sem. Fac. Sci. Univ. Cagliari 46 (1976) 81-88. 
[8] M. Meier, Boundedness and integrability properties of weak solutions of quasilinear elliptic systems, J. Reine Angew. Math. 333 (1982) 191-220.

[9] G. Mingione, Regularity of minima: an invitation to the dark side of the calculus of variations, Appl. Math. 51 (2006) $355-426$

[10] G. Mingione, Singularities of minima: a walk on the wild side of the calculus of variations, J. Global Optim. 40 (2008) 209-223.

[11] C. Mooney, O. Savin, Some singular minimizers in low dimensions in the calculus of variations, Arch. Ration. Mech. Anal. 221 (2016) 1-22.

[12] J. Nečas, J. Stará, Principio di massimo per i sistemi ellittici quasi-lineari non diagonali, Boll. Unione Mat. Ital. (4) 6 (1972) $1-10$. 